\title{
Optimal doses of caspofungin during continuous venovenous hemodiafiltration in critically ill patients
}

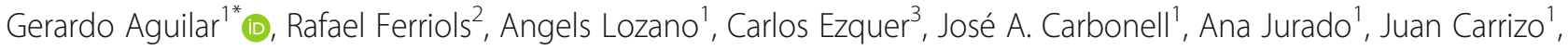
Ferran Serralta', Jaume Puig ${ }^{1}$, David Navarro ${ }^{4,5}$, Manuel Alos $^{2}$ and F. Javier Belda ${ }^{1,5}$

Keywords: Echinocandins, Continuous renal replacement therapy, Invasive candidiasis

The aim of the present study was to describe the pharmacokinetics of caspofungin in 12 critically ill adult patients with suspected or proven invasive candidiasis who were receiving continuous venovenous hemodiafiltration (CVVHD).

CVVHD was performed using a polysulfone hemofilter (Fresenius, Germany). Caspofungin was administered at usual doses. Pre-filter and post-filter blood, ultradiafiltrate, and urine samples were collected at steady state on day 3 or later, before the dose infusion started, and $0.5,1,1.5,2,4,6,8$, and $24 \mathrm{~h}$ after the infusion ended.

The drug concentrations were measured by high performance liquid chromatograpy (HPLC) and the following pharmacokinetic parameters were calculated: area under the concentration-time curve $\left(\mathrm{AUC}_{0-24 \mathrm{~h}}\right)$, elimination $\mathrm{t}_{1 / 2}$, volume of distribution $(\mathrm{Vd})$, clearance, trough concentration $\left(\mathrm{C}_{\text {trough }}\right)$, and maximum concentration $\left(\mathrm{C}_{\text {max }}\right)$.

The results of our study are summarized in Tables 1 and 2 and Fig. 1. Caspofungin was negligible in the ultradiafiltrate and urine samples, confirming the lack of drug elimination through hemofiltration or hemodialysis. Similar findings were previously described by Weiler et al. [1]. Additionally, the mean concentration of caspofungin was slightly higher in the post-filter line than in the pre-filter line (Fig. 1), allowing us to rule out the adsorption to the filter hypothesized in other studies with echinocandins $[2,3]$.

In four patients (33\%), the trough concentration of caspofungin was lower than the $\mathrm{MIC}_{90} \mathrm{~s}$ published for Candida and Aspergillus spp., including Candida parapsilosis (2 mg/L) [4]. On the other hand, among echinocandins, micafungin has been associated with $1 \log$ kill/24 $\mathrm{h}$ in a murine model of disseminated candidiasis when an AUC/MIC of 865, 450, or 1185 is achieved for Candida albicans, Candida glabrata, or C. parapsilosis, respectively [5]. Taking into account a MIC of $0.1 \mathrm{mg} / \mathrm{L}$ [4], and using the target pharmacokinetics/pharmacodynamics (PK/PD) described for micafungin, we would have reached this concentration in only nine patients $(75 \%$, AUC $>86.5 \mathrm{mg} \mathrm{h} / \mathrm{L}$ ) for C. albicans and four patients (33\%, AUC > $118.5 \mathrm{mg} \mathrm{h} / \mathrm{L}$ ) for C. parapsilosis but all patients for C. glabrata (AUC $>45 \mathrm{mg} \mathrm{h} / \mathrm{L}$ ) (Table 2). These data suggest that caspofungin dosing could be insufficient in some critically ill patients.

In conclusion, CVVHD appears to have a negligible effect on caspofungin clearance. However, the licensed regimen of caspofungin was not adequate to reach the PK/PD targets in some critically ill patients, regardless of the use of CVVHD. Nevertheless, future studies are needed to confirm these findings.

\footnotetext{
* Correspondence: gerardo.aguilar@uv.es

'Surgical Intensive Care Unit, Department of Anesthesiology and Intensive Care, Hospital Clínico Universitario de Valencia, Avenida Blasco Ibáñez, 17, 46010 Valencia, Spain

Full list of author information is available at the end of the article
} 
Table 1 Individual arterial caspofungin concentrations (mg/L) of the 12 patients studied

\begin{tabular}{|c|c|c|c|c|c|c|c|c|c|c|c|c|}
\hline Time (h) & 1 & 2 & 3 & 4 & 5 & 6 & 7 & 8 & 9 & 10 & 11 & 12 \\
\hline Predose & 3.09 & 2.12 & 2.94 & 0.90 & 1.50 & 3.04 & 2.10 & 2.93 & 2.18 & 3.16 & 2.69 & 2.62 \\
\hline 0.5 & 10.85 & 6.96 & 8.50 & 4.38 & 4.59 & 9.86 & 7.09 & 8.23 & 7.81 & 11.17 & 10.24 & 7.02 \\
\hline 1 & 9.34 & 6.19 & 8.23 & 2.80 & 4.44 & 9.11 & 6.10 & 7.23 & 6.69 & 9.91 & 8.88 & 5.78 \\
\hline 1.5 & 8.55 & 5.75 & 7.05 & NA & 4.41 & 8.24 & 5.27 & 6.04 & 6.03 & 8.42 & 8.39 & 5.09 \\
\hline 2 & 7.51 & 5.47 & 6.91 & 2.43 & 3.85 & 7.37 & 4.96 & 5.88 & 5.72 & 7.74 & 7.92 & 4.61 \\
\hline 4 & 6.38 & 4.49 & 6.13 & 2.12 & 3.77 & 6.54 & 4.13 & 5.66 & 5.32 & 6.94 & 6.62 & 3.94 \\
\hline 6 & 5.63 & 3.96 & 5.63 & NA & 3.04 & 5.84 & 3.54 & 5.33 & 4.55 & 6.40 & 6.31 & 3.60 \\
\hline 8 & 5.00 & 3.40 & 5.22 & 1.99 & 2.80 & 4.71 & 3.10 & 4.45 & 4.49 & 5.61 & 6.00 & 3.27 \\
\hline 24 & 3.47 & 2.30 & 3.10 & 1.34 & 1.59 & 2.47 & 1.63 & 2.73 & 2.27 & 2.88 & 4.00 & 1.85 \\
\hline
\end{tabular}

Time refers to the time since caspofungin infusion ended. NA data not available

Table 2 Pharmacokinetics of caspofungin during continuous venovenous hemodiafiltration in 12 patients

\begin{tabular}{|c|c|c|c|c|c|c|c|c|}
\hline \multirow[b]{2}{*}{ Patient } & \multicolumn{3}{|c|}{$\mathrm{AUC}_{0-24}(\mathrm{mg} \mathrm{h} / \mathrm{L})$} & \multirow[b]{2}{*}{$\overline{V d}(\mathrm{~L})$} & \multirow[b]{2}{*}{$\mathrm{Cl}(\mathrm{L} / \mathrm{h})$} & \multirow[b]{2}{*}{$C_{\max }(\mathrm{mg} / \mathrm{L})$} & \multirow[b]{2}{*}{$C_{\text {trough }}(\mathrm{mg} / \mathrm{L})$} & \multirow[b]{2}{*}{$t_{1 / 2}(h)$} \\
\hline & Arterial & Venous & $\begin{array}{l}\text { Difference venous } \\
\text { to arterial (\%) }\end{array}$ & & & & & \\
\hline 1 & 140.0 & 180.0 & 29 & 14.1 & 0.356 & 12.5 & 3.47 & 27.4 \\
\hline 2 & 88.3 & 106.0 & 20 & 17.1 & 0.567 & 7.8 & 2.1 & 21.0 \\
\hline 3 & 124.0 & 152.0 & 23 & 10.9 & 0.402 & 8.8 & 3.1 & 18.8 \\
\hline 4 & 65.4 & 77.4 & 18 & 26.8 & 0.765 & 6.9 & 1.3 & 24.3 \\
\hline 5 & 68.0 & 90.0 & 32 & 17.5 & 0.735 & 4.8 & 1.5 & 16.5 \\
\hline 6 & 102.0 & 107.0 & 5 & 13.6 & 0.683 & 10.7 & 2.5 & 13.8 \\
\hline 7 & 65.6 & 78.8 & 20 & 15.0 & 0.762 & 8.3 & 1.6 & 13.6 \\
\hline 8 & 100.0 & 113.0 & 13 & 13.9 & 0.499 & 9.5 & 2.7 & 19.3 \\
\hline 9 & 102.0 & 127.0 & 25 & 14.1 & 0.685 & 9.2 & 2.3 & 14.3 \\
\hline 10 & 121.0 & 142.0 & 17 & 12.5 & 0.578 & 12.6 & 2.9 & 15.0 \\
\hline 11 & 190.0 & 224.0 & 18 & 13.9 & 0.368 & 11.5 & 4.0 & 26.2 \\
\hline 12 & 60.1 & 74.5 & 24 & 27.7 & 1.165 & 8.5 & 1.9 & 16.5 \\
\hline Mean \pm SD & $102 \pm 46$ & $123 \pm 46$ & $20.3 \pm 7.2$ & $16.4 \pm 5.4$ & $0.630 \pm 0.225$ & $9.3 \pm 2.3$ & $2.4 \pm 0.8$ & $18.9 \pm 4.9$ \\
\hline
\end{tabular}

\section{$S D$ standard deviation}

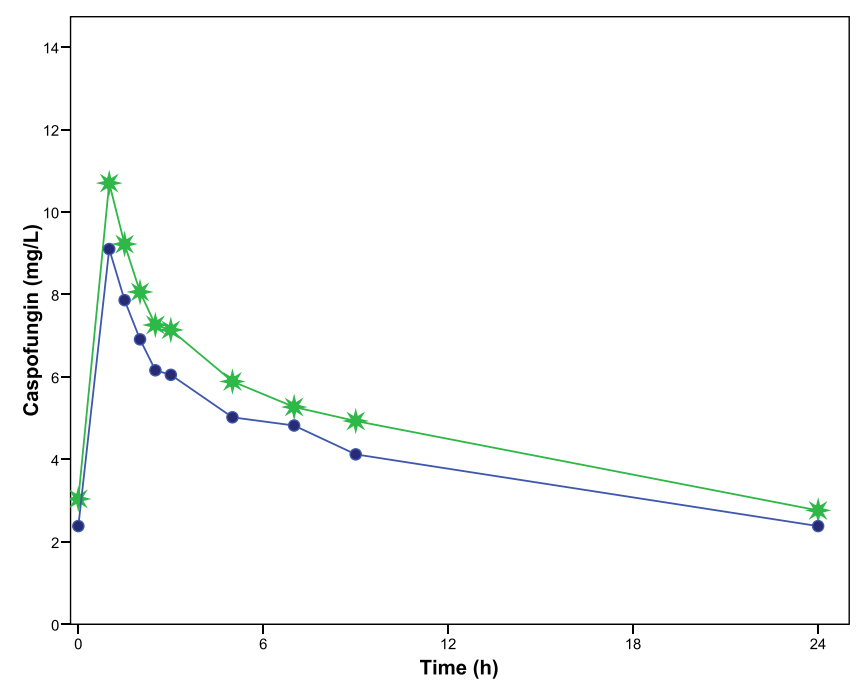

Fig. 1 Average caspofungin concentration over time. Infusion started at $0 \mathrm{~h}$ and continued over 1 h. $n=12$ patients. Solid dots, arterial; asterisks, venous. (The figure is original for this article) 


\section{Abbreviations}

AUC: Area under the concentration-time curve; $C_{\text {max }}$ : Maximum concentration; $C_{\text {trough: }}$ Trough concentration; CWHD: Continuous venovenous hemodiafiltration; MIC 90 : Minimum inhibitory concentration required to inhibit the growth of $90 \%$ of a microorganism; PK/PD: pharmacokinetic/pharmacodynamic; Vd: Volume of distribution

\section{Acknowledgements}

Not applicable.

\section{Funding}

The study was funded by MSD Spain.

\section{Availability of data and materials}

All relevant data are within the paper and its supporting information files. All data are fully available without restriction.

\section{Authors' contributions}

GA conceived the study, participated in its design, and drafted the manuscript. RF participated in designing and coordinating the study and helped to draft the manuscript. CE carried out the pharmacokinetics analysis and helped to revise the manuscript. $A L, J A C, A J, J C$, and FS participated in analyzing and interpreting the data and helped to revise the manuscript. JP, DN, MA, and FJB participated in the design and coordination of the study and revised the manuscript. All authors read and approved the final manuscript.

\section{Competing interests}

G.A. received funds for speaking at meetings organized on behalf of Astellas, Gilead, Merck Sharp and Dohme (MSD), and Pfizer, as well as unrestricted research grants from Astellas, MSD, and Pfizer. D.N. received funds for speaking at meetings organized on behalf of Astellas, MSD, and Pfizer and received unrestricted research grants from Astellas and Pfizer. All other authors declare no competing interests.

\section{Consent for publication}

Written informed consent was obtained from the patients or their relatives for publication of their individual details. The consent form is held by the authors' institution and is available for review by the Editor-in-Chief.

\section{Ethics approval and consent to participate}

The study protocol (MER-CAS-2013-01) was approved by the local ethics committee (Instituto de Investigación Sanitaria, INCLIVA) and written informed consent obtained from the patients or their relatives prior to study inclusion.

\section{Author details}

${ }^{1}$ Surgical Intensive Care Unit, Department of Anesthesiology and Intensive Care, Hospital Clínico Universitario de Valencia, Avenida Blasco Ibáñez, 17, 46010 Valencia, Spain. ${ }^{2}$ Department of Pharmacy, Hospital Clínico Universitario de Valencia, Avenida Blasco Ibáñez, 17, 46010 Valencia, Spain. ${ }^{3}$ Instituto de Investigación Sanitaria, INCLIVA, Avenida Blasco Ibáñez, 17, 46010 Valencia, Spain. ${ }^{4}$ Department of Microbiology, Hospital Clínico

Universitario de Valencia, Avenida Blasco Ibáñez, 17, 46010 Valencia, Spain. ${ }^{5}$ School of Medicine, University of Valencia, Avenida Blasco Ibáñez, 15, 46010 Valencia, Spain.

Published online: 30 January 2017

\section{References}

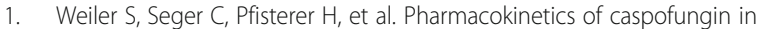
critically ill patients on continuous renal replacement therapy. Antimicrob Agents Chemother. 2013;57(8):4053-7.

2. Leitner JM, Meyer B, Fuhrmann V, et al. Multiple-dose pharmacokinetics of anidulafungin during continuous venovenous haemofiltration. J Antimicrob Chemother. 2011;66(4):880-4

3. Gonzalez de Molina F, Martinez-Alberici Mde L, Ferrer R. Treatment with echinocandins during continuous renal replacement therapy. Crit Care. 2014;18(2):218.
4. Chen SC, Slavin MA, Sorrell TC. Echinocandin antifungal drugs in fungal infections: a comparison. Drugs. 2011;71:11-41.

5. Andes D, Ambrose PG, Hammel JP, et al. Use of pharmacokineticpharmacodynamic analyses to optimize therapy with the systemic antifungal micafungin for invasive candidiasis or candidemia. Antimicrob Agents Chemother. 2011;55(5):2113-21. 